\title{
Multiband Transit Light Curve Modeling of WASP-4
}

\author{
N. Nikolov ${ }^{1}$, J. Koppenhoefer ${ }^{2,3}$, M. Lendl ${ }^{4}$, \\ T. Henning ${ }^{1}$ and J. Greiner ${ }^{3}$ \\ ${ }^{1}$ Max-Planck-Institut für Astronomie, Königstuhl 17, 69117 Heidelberg, Germany \\ email: nikolov@mpia.de \\ ${ }^{2}$ Universitäts-Sternwarte München, Scheinerstr. 1, 81679 Munich, Germany \\ ${ }^{3}$ Max Planck Institute for Extraterrestrial Physics, Geissenbachstr., 85748 Garching, Germany \\ ${ }^{4}$ Observatoire de Genève, Universitè de Genève, 51 chemin des Maillettes, 1290 Sauverny, \\ Switzerland
}

\begin{abstract}
We report on the simultaneous $g^{\prime}, r^{\prime}, i^{\prime}, z^{\prime}$ multiband, high time sampling (18-24s) ground-based photometric observations, which we use to measure the planetary radius and orbital inclination of the extrasolar transiting hot Jupiter WASP-4b. We recorded 987 images during three complete transits with the GROND instrument, mounted on the MPG/ESO-2.2m telescope at La Silla Observatory. Assuming a quadratic law for the stellar limb darkening we derive system parameters by fitting a composite transit light curve over all bandpasses simultaneously. To compute uncertainties of the fitted parameters we employ the Bootstrap Monte Carlo Method. The three central transit times are measured with precision down to $6 \mathrm{~s}$. We find a planetary radius $R_{p}=1.413 \pm 0.020 R_{\mathrm{Jup}}$, an orbital inclination $i=88 .^{\circ} 57 \pm 0.45^{\circ}$ and calculate new ephemeris, a period $P=1.33823144 \pm 0.00000032$ days and reference transit epoch $T_{0}=2454697.798311 \pm 0.000046$ (BJD). The analysis of the new transit mid-times in combination with previous measurements imply a constant orbital period and no compelling evidence for TTVs due to additional bodies in the system.
\end{abstract}

Keywords. planetary systems, stars: individual (WASP-4b)

\section{Introduction}

Exoplanetary transits provide a direct access to some of the most interesting physical parameters of these objects. Currently, the transit method is the only tool to measure accurate planetary radii, orbital inclinations, and mean planetary densities, if the radius and the mass of the parent star are known. In this work we aimed at obtaining high precision multiband photometry during three transits of WASP-4b with the ultimate goals to (i) measure accurate system parameters and (ii) to obtain times of minimum light, which we use to refine the transit ephemeris and to search for transit timing variations (TTVs).

\section{Observations and analysis}

We observed three transits of WASP-4b (Wilson et al. 2008) on UT August 26 and 30 and October 8, 2009 using the Gamma Ray Burst Optical and Near-Infrared Detector (GROND), mounted on the MPG/ESO-2.2m telescope at ESO La Silla Observatory (see Fig. 1). We perform circular aperture photometry of 987 calibrated science images. The best apertures are selected by minimizing the magnitude root-mean-square of the out of transit data. We improve the quality of the WASP-4 transit light curves by modeling 


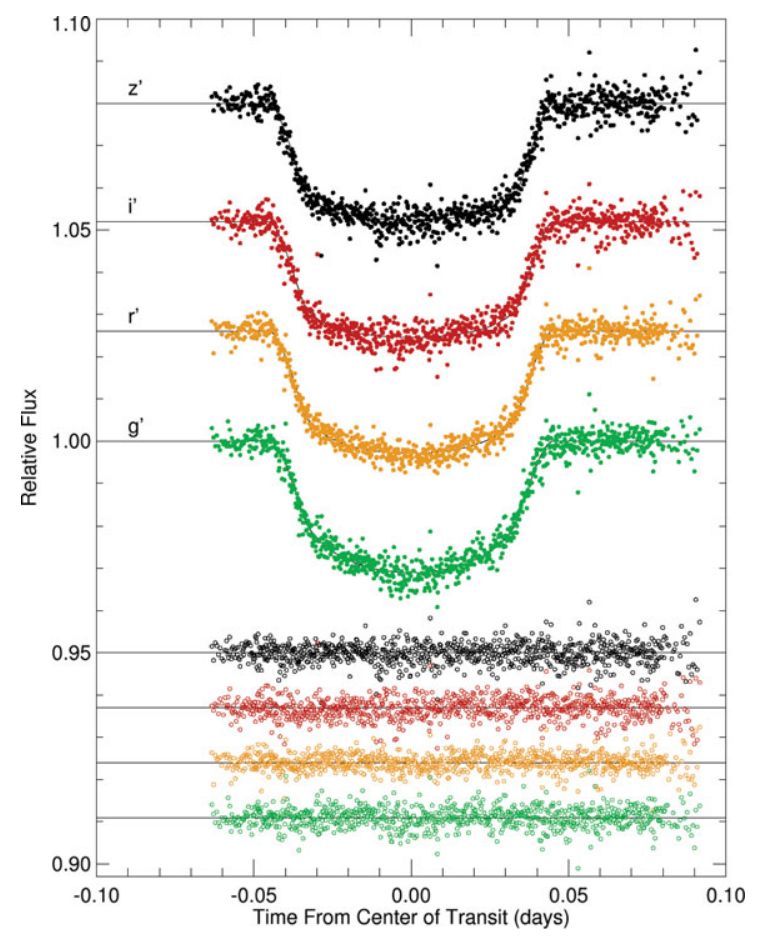

Figure 1. GROND composite transit photometry of WASP-4b, acquired on August 26, August 30 and October 8, 2009. The light curves of each band are shifted with 0.026 in relative flux for clarity. The best-fit transit models are displayed with continuous lines and the residuals from the best-fit modeling are shown at bottom.

the data for two effects: (i) differential air-mass extinction and (ii) correlation of the magnitude with the $\mathrm{x}, \mathrm{y}$ position of the psf on the detector.

We derive the best-fit transit parameters (mid-transit time, $T_{0}$, orbital period, $P$, planet to star radius ratio, $R_{p} / R_{*}$, normalized semimajor axis, $a / R_{*}$ and orbital inclination, $i$ ) using the light curve models of Mandel \& Agol (2002) and minimizing the $\chi^{2}$ function over the four passbands simultaneously via the down-hill simplex algorithm (Press et al. 1992). To compute uncertainties for the fitted parameters we employ the Bootstrap Monte Carlo method (Press et al. 1992), taking into account time-correlated noise by (i) rescaling the photometric weights, so that the best-fitting model for each band and run results in a reduced $\chi^{2}=1$ and (ii) by following the "time-averaging" method described in Pont et al. (2006).

We also investigated the observed minus calculated $(O-C)$ residuals of our data and the reported transit mid-times at the time of writing for any departures from the predicted values estimated using our ephemeris. We find no compelling evidence for transit timing variations due to additional bodies in the system.

\section{References}

Mandel, K. \& Agol, E. 2002, ApJ, 580L, 171

Pont, F., Zucker, S., \& Queloz, D. 2006, MNRAS, 373, 231

Press, W. H., Teukolsky, S. A., Vetterling, W. T., \& Flannery, B. P. 1992, Numerical recipes in C. The art of scientific computing, ed. T.S.A.V.W.T.F.B.P. Press, W. H.

Wilson, D. M., Gillon, M., Hellier, C., et al., 2008, ApJ, 675, 113 